\title{
Relaxation due to random collisions with a many-qudit environment
}

\author{
Giuseppe Gennaro, ${ }^{1}$ Giuliano Benenti, ${ }^{2,3}$ and G. Massimo Palma ${ }^{4}$ \\ ${ }^{1}$ Dipartimento di Scienze Fisiche ed Astronomiche, Università di Palermo, via Archirafi 36, I-90123 Palermo, Italy \\ ${ }^{2}$ CNISM, CNR-INFM, and Center for Nonlinear and Complex systems, Università degli Studi dell'Insubria, \\ via Valleggio 11, I-22100 Como, Italy \\ ${ }^{3}$ Sezione di Milano, Istituto Nazionale di Fisica Nucleare, via Celoria 16, I-20133 Milano, Italy \\ ${ }^{4}$ NEST-CNR (INFM) and Dipartimento di Scienze Fisiche ed Astronomiche, Università di Palermo, \\ via Archirafi 36, I-90123 Palermo, Italy \\ (Received 21 November 2008; published 5 February 2009)
}

\begin{abstract}
We analyze the dynamics of a system qudit of dimension $\mu$ sequentially interacting with the $\nu$-dimensional qudits of a chain playing the role of an environment. Each pairwise collision has been modeled as a random unitary transformation. The relaxation to equilibrium of the purity of the system qudit, averaged over random collisions, is analytically computed by means of a Markov chain approach. In particular, we show that the steady state is the one corresponding to the steady state for random collisions with a single environment qudit of effective dimension $\nu_{e}=\nu \mu$. Finally, we numerically investigate aspects of the entanglement dynamics for qubits $(\mu=\nu=2)$ and show that random unitary collisions can create multipartite entanglement between the system qudit and the qudits of the chain.
\end{abstract}

DOI: 10.1103/PhysRevA.79.022105

PACS number(s): 03.65.Yz, 03.67.Mn

\section{INTRODUCTION}

The repeated collision model is a simple yet instructive model of irreversible quantum dynamics. First introduced in [1] to analyze the process of thermalization and more generally of homogenization [2-4] in the limit of an environment with a large number of degrees of freedom, it has been further studied to elucidate various aspects of the irreversible dynamics of quantum systems in the presence of environments with few degrees of freedom [5,6]. In most of the literature cited above, the interaction between system and environment is due to pairwise elastic collisions modeled by partial SWAP operators. In the present paper we will instead model such collisions by random unitary operators [7-12]. This choice is due to several reasons. On the one hand, although an exact modeling of the system-environment interaction is difficult, a good description of the relaxation process can be obtained by a suitable average over random interactions. Some examples of such an approach can be found in $[13,14]$, where the irreversible dynamics of a single and a pair of qubits is analyzed in terms of a random interaction with an environment which is itself modeled as a random matrix, and in [6], where the average dynamics of a single qubit interacting with a very small reservoir is described again by random collisions. On the other hand, random and pseudorandom states and their efficient generation by suitable sequences of random gates [15-24] have received considerable attention due to possible applications in quantum-information processing [25-28].

In the present paper, we analyze the approach to equilibrium of a system qudit interacting with a very large ensemble of qudits. The interaction is modeled by a sequence of twoqudit random collisions. The paper is structured as follows. In the following section we review the random collision model and we specialize it to the case of collisions described by random unitary operators. We then characterize the approach to equilibrium by analytically calculating the purity of the system steady state and the rate of approach to such a state for the specific case of colliding qubits. This analytical analysis is then generalized to a system qudit of dimension $\mu$ colliding with an ensemble of qudits of size $\nu$. We then proceed with a numerical analysis of the entanglement dynamics for colliding qubits.

\section{THE RANDOM COLLISION MODEL AND THE RELAXATION TO EQUILIBRIUM}

In the random collisions model of irreversible dynamics a system qudit interacts with an environment consisting of $N$ qudits. Such interaction is modeled by pairwise collisions between the system qudit and a single environment qudit. Each collision is described by a random unitary operator. The environment, i.e., $N$, is assumed to be so large that the system never collides twice with the same environment qudit. In pictorial terms one can think of a single qudit colliding in sequence with the individual qudits of a long chain. The overall state of the system and environment, after $t$ collisions, is

$$
\varrho_{S E}^{(t)}=U_{0 t} \cdots U_{02} U_{01} \varrho_{S E}^{(0)} U_{01}^{\dagger} U_{02}^{\dagger} \cdots U_{0 t}^{\dagger},
$$

where $U_{0 j}$ is a random unitary operator acting on the pair of qudits $0, j ; 0$ labels the system qudit and $j=1, \ldots, N$ labels the environment qudits. Let us assume that the system and environment are in an initial tensor product state $\varrho_{S E}^{(0)}$ $=\varrho_{S}^{(0)} \eta_{1} \eta_{2} \cdots \eta_{N}$. Since the collision operators are random unitaries, the specific states $\eta_{i}$ are irrelevant, as long as they have the same purity, as will be explained shortly, and we can assume that all the environment qudits are in the same initial state $\eta$.

In order to characterize the relaxation process we first consider the decay of the system purity after $t$ collisions. We remind the reader that, given a density operator $Q$, its purity is defined as $P=\operatorname{Tr}\left[\varrho^{2}\right]$. The purity is a decreasing function of the degree of statistical mixture of $\varrho$ and, for a qudit of 
dimension $\mu$ takes values in the range $1 / \mu \leqslant P \leqslant 1$, where $P=1$ corresponds to pure states and $P=1 / \mu$ to the completely unpolarized mixed state. Since we are focusing our attention on the purity of the system, our model is equivalent to a system qudit colliding with a single environment qudit whose state is refreshed to its initial state $\eta$ after each collision. We will show that, after averaging over random unitary collisions, $\mathcal{P}(t)$ can be analytically calculated (hence after we will use the symbol $\mathcal{P}$ to mean the analytic ensemble average value of $P$ ).

\section{A. Colliding qubits}

Let us first consider the case in which both system and environment consist of qubits. The density operator of the system and environment qubits can be written as

$$
\varrho_{S E}=\sum_{\alpha_{0}, \alpha_{E}} c_{\alpha_{0} \alpha_{E}} \sigma_{0}^{\alpha_{0}} \otimes \sigma_{E}^{\alpha_{E}},
$$

where $\sigma_{0}^{\alpha_{0}}\left(\sigma_{E}^{\alpha_{E}}\right)$ denotes a Pauli matrix acting on the system (environment) qubit, with $\alpha_{i} \in\{0, x, y, z\}$ and $\sigma^{0}=I$. The purity of the overall system and environment after $t$ collisions then reads

$$
P_{S E}=4 \sum_{\alpha_{0} \alpha_{1}} c_{\alpha_{0} \alpha_{E}}^{2}(t)
$$

and the system's purity is given by

$$
P(t)=8 \sum_{\alpha_{0}} c_{\alpha_{0} 0}^{2}(t)
$$

Note that $P_{S E}$ is invariant under unitary evolution, i.e., the overall system-environment purity is the same before and after each collision (of course before the state of the environment qubit is refreshed). The constraints

$$
\operatorname{Tr}\left(\varrho_{S E}\right)=1, \quad \operatorname{Tr}\left(\varrho_{S E}^{2}\right)=P_{S E}
$$

lead to

$$
c_{00}=\frac{1}{4}, \quad \sum_{\left(\alpha_{0}, \alpha_{1}\right) \neq(0,0)} c_{\alpha_{0} \alpha_{1}}^{2}=\frac{4 P_{S E}-1}{16} .
$$

It has been shown [21] that when two qubits collide with a sequence of random U(4) unitaries, the ensemble-averaged coefficients $c^{2}(t)$ evolve according to a Markov chain as

$$
c^{2}(t+1)=c^{2}(t) M,
$$

where

$$
c^{2}=\left(c_{00}^{2}, c_{0 x}^{2}, \ldots, c_{z z}^{2}\right)
$$

and

$$
M=\left(\begin{array}{cccc}
1 & 0 & \cdots & 0 \\
0 & \frac{1}{15} & \cdots & \frac{1}{15} \\
\vdots & \vdots & \ddots & \vdots \\
0 & \frac{1}{15} & \cdots & \frac{1}{15}
\end{array}\right)
$$

is a Markov $16 \times 16$ matrix. The structure of $M$ is clear: it leaves $c_{00}^{2}$ unchanged while all the other components of $c^{2}$ are uniformly mixed. The equilibrium state of such a chain, if the state of the environment qubit is not refreshed after each collision, must lie in the subspace spanned by the eigenvectors of $M$ with eigenvalue 1 . A normalized basis corresponding to the unit eigenvalue of $M$ is given by

$$
v_{0}=(1,0, \ldots, 0), \quad v_{1}=\frac{1}{\sqrt{15}}(0,1, \ldots, 1) .
$$

The equilibrium state then reads

$$
v=x_{0} v_{0}+x_{1} v_{1}
$$

where

$$
\begin{gathered}
x_{0}=\left\langle v_{0}, v\right\rangle=c_{00}^{2}=\frac{1}{16}, \\
x_{1}=\left\langle v_{1}, v\right\rangle=\frac{1}{\sqrt{15}} \sum_{\left(\alpha_{0}, \alpha_{1}\right) \neq(0,0)} c_{\alpha_{0} \alpha_{1}}^{2}=\frac{1}{\sqrt{15}} \frac{4 \mathcal{P}_{S E}-1}{16} .
\end{gathered}
$$

Therefore,

$$
v=\left(\frac{1}{16}, \frac{4 \mathcal{P}_{S E}-1}{240}, \ldots, \frac{4 \mathcal{P}_{S E}-1}{240}\right) .
$$

Finally, we obtain, for the ensemble-averaged purity

$$
\mathcal{P}=8 \sum_{\alpha_{0}} c_{\alpha_{0} 0}^{2}=\frac{1}{2}+\frac{1}{10}\left(4 \mathcal{P}_{S E}-1\right) .
$$

For pure system-environment states $\left(\mathcal{P}_{S E}=1\right)$, we recover Lubkin's result [29]

$$
\mathcal{P}=\mathcal{P}_{L}=\frac{4}{5}
$$

It is important to note that such (ensemble averaged) value of purity is reached after a single random collision. In other words, as it should be, the state of the system and of the first environment qubit after a single collision is a two-qubit random state.

We now consider the case in which the state of the environment qubit is refreshed after each collision. After $t$ collisions Eq. (13) becomes

$$
\mathcal{P}(t)=\frac{1}{2}+\frac{1}{10}\left[4 \mathcal{P}_{S E}(t-1)-1\right] \quad(t=1,2, \ldots) .
$$

Note, however, that $\mathcal{P}_{S E}$ changes when the environment qubit is reset to its initial state $\eta$. Just before the $t$ th collision we have

$$
\mathcal{P}_{S E}(t-1)=\mathcal{P}(t-1) P_{\eta},
$$

where $P_{\eta}$ is the purity of the unperturbed environment qubit state $\eta$.

We now solve the equation

$$
\mathcal{P}(t)=\frac{1}{2}+\frac{1}{10}\left[4 \mathcal{P}(t-1) P_{\eta}-1\right] \quad(t=1,2, \ldots) .
$$

The equilibrium value 


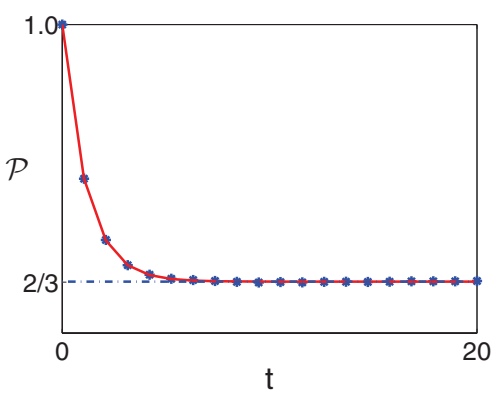

FIG. 1. (Color online) Ensemble-averaged purity functional behavior for $\mu=2, \nu=2$. The limit value is $\mathcal{P}(\infty)=2 / 3$. The markers are the ensemble-averaged values. The curve shows the analytic result of Eq. (22).

$$
\mathcal{P}(\infty)=\frac{2}{5-2 P_{\eta}}
$$

is obtained as the solution to the equation

$$
\mathcal{P}(\infty)=\frac{1}{2}+\frac{1}{10}\left[4 \mathcal{P}(\infty) P_{\eta}-1\right]
$$

If we define $\xi(t)=\mathcal{P}(t)-\mathcal{P}(\infty)$, we obtain

$$
\xi(t)=\frac{2}{5} P_{\eta} \xi(t-1)=\left(\frac{2}{5} P_{\eta}\right)^{t} \xi(0) .
$$

Note that $\mathcal{P}(\infty)$ as well as the decay rate to equilibrium depend on $\mathcal{P}_{\eta}$ : the lowest the purity of the environment qubits the fastest is the approach to equilibrium and the lowest the equilibrium value of the system purity. Although this has some analogies with the relaxation to thermal equilibrium it is worth stressing that the two processes have some important differences. In particular note that, contrary to the case of thermalization, $\mathcal{P}(\infty) \neq \mathcal{P}_{\eta}$. Furthermore, the dependence on $\mathcal{P}_{\eta}$ of the decay rate is also not the one expected for the relaxation to equilibrium. This is not surprising as collisions modeled by random collisions are not elastic. Indeed it has been shown in [1] that the only operator guaranteeing thermalization (and in general homogenization [2]) is the partial swap.

When the chain qubits are initially in a pure state, we have

$$
\mathcal{P}(\infty)=\frac{2}{3}
$$

and Eq. (20) becomes

$$
\mathcal{P}(t)=\left(\frac{2}{5}\right)^{t}\left(\mathcal{P}(0)-\frac{2}{3}\right)+\frac{2}{3}=e^{-\lambda t}\left(\mathcal{P}(0)-\frac{2}{3}\right)+\frac{2}{3},
$$

where $\lambda=\ln (5 / 2)=0.916 \ldots$ is the rate of approach to equilibrium. Starting from a pure state we obtain $\mathcal{P}(1)=4 / 5$, $\mathcal{P}(2)=54 / 75=0.72, \mathcal{P}(3)=258 / 375 \approx 0.688, \ldots$, in agreement with our numerical data, as shown in Fig. 1.

\section{B. Colliding qudits}

The above Markov chain technique can be generalized to analyze the more general case in which a system qudit of dimension $\mu$ collides with a large number of environment qudits of dimension $\nu$. In this case system-environment interactions are modeled by random unitaries drawn from the $\mathrm{U}(L)$-invariant Haar measure in the overall Hilbert space of size $L=\mu \nu$. The steps of the analysis previously carried on for qubits can be straightforwardly followed to obtain the equilibrium value and the decay rate of the purity of colliding qudits. We use again representation (2), where the $\sigma$ 's are now generalized Pauli matrices [30,31]. The purity of the overall system then reads

$$
P_{S E}=\mu \nu \sum_{\alpha_{0}=0}^{\mu-1} \sum_{\alpha_{1}=0}^{\nu-1} c_{\alpha_{0} \alpha_{1}}^{2}(t)
$$

and the system's purity is given by

$$
P(t)=\mu \nu^{2} \sum_{\alpha_{0}} c_{\alpha_{0} 0}^{2}(t) .
$$

The constraints

$$
\operatorname{Tr}\left(\varrho_{S E}\right)=1, \quad \operatorname{Tr}\left(\varrho_{S E}^{2}\right)=P_{S E}
$$

lead to

$$
c_{00}(t)=\frac{1}{\mu \nu}, \quad \sum_{\left(\alpha_{0}, \alpha_{1}\right) \neq(0,0)} c_{\alpha_{0} \alpha_{1}}^{2}(t)=\frac{\mu \nu P_{S E}-1}{(\mu \nu)^{2}} .
$$

Also in this case the vector

$$
c^{2}=\left(c_{00}^{2}, \cdots, c_{\mu \nu}^{2}\right)
$$

evolves in time according to a Markov chain, as in Eq. (7). We extend Znidarič's conjecture [21] about the form of the Markov matrix by assuming again that $M$ leaves $c_{00}^{2}$ unchanged while it uniformly mixes all other components. $M$ must therefore be a $(\mu \nu) \times(\mu \nu)$ matrix of the form

$$
M=\left(\begin{array}{cccc}
1 & 0 & \cdots & 0 \\
0 & \frac{1}{(\mu \nu)^{2}-1} & \cdots & \frac{1}{(\mu \nu)^{2}-1} \\
\vdots & \vdots & \ddots & \vdots \\
0 & \frac{1}{(\mu \nu)^{2}-1} & \cdots & \frac{1}{(\mu \nu)^{2}-1}
\end{array}\right) .
$$

The two-dimenisonal eigenspace corresponding to the unit eigenvalue of $M$ is spanned by the vector basis

$$
v_{0}=(1,0, \ldots, 0), \quad v_{1}=\frac{1}{\sqrt{(\mu \nu)^{2}-1}}(0,1, \ldots, 1) .
$$

Following the same steps that lead to Eq. (12) we obtain the equilibrium state as

$$
v=\left(\frac{1}{(\mu \nu)^{2}}, \frac{\mu \nu \mathcal{P}_{S E}-1}{(\mu \nu)^{2}\left[(\mu \nu)^{2}-1\right]}, \ldots, \frac{\mu \nu \mathcal{P}_{S E}-1}{(\mu \nu)^{2}\left[(\mu \nu)^{2}-1\right]}\right) .
$$

The system purity of this state is 


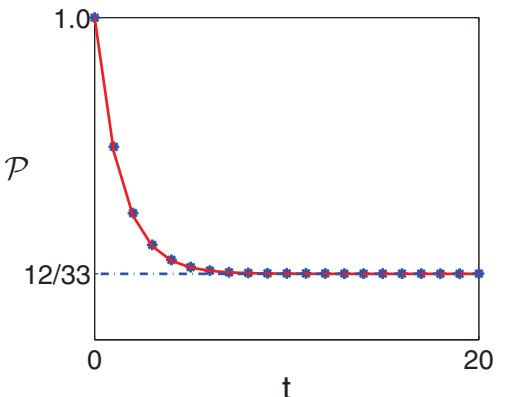

FIG. 2. (Color online) Ensemble-averaged purity functional behavior for $\mu=4, \nu=2$. The limit value is $\mathcal{P}(\infty)=12 / 33$. The markers are the ensemble-averaged values. The curve shows the analytic result of Eq. (37)

$$
\mathcal{P}=\mu \nu^{2} \sum_{\alpha_{0}} c_{\alpha_{0} 0}^{2}=\frac{1}{\mu}+\frac{\mu^{2}-1}{\mu\left[(\mu \nu)^{2}-1\right]}\left(\mu \nu \mathcal{P}_{S E}-1\right) .
$$

If the initial system-environment state is pure $\left(\mathcal{P}_{S E}=1\right)$, we recover Lubkin's result [29]

$$
\mathcal{P}=\frac{\mu+\nu}{\mu \nu+1} .
$$

This means again that after a single collision the state of the system and of the first colliding qudit is a random state in the Hilbert space of dimension $\mu \otimes \nu$.

If the environment is refreshed after each collision, we have

$$
\mathcal{P}(t+1)=\frac{1}{\mu}+\frac{\mu^{2}-1}{\mu\left[(\mu \nu)^{2}-1\right]}[\mu \nu \mathcal{P}(t)-1] \quad(t=0,1, \ldots) .
$$

This equation leads to the following steady value of the purity:

$$
\mathcal{P}(\infty)=\frac{\mu+(\mu \nu)}{\mu(\mu \nu)+1} .
$$

If we define $\xi(t)=\mathcal{P}(t)-\mathcal{P}(\infty)$, we obtain

$$
\xi(t)=\alpha \xi(t)=\alpha^{t} \xi(0),
$$

where

$$
\alpha \equiv \frac{\nu\left(\mu^{2}-1\right)}{(\mu \nu)^{2}-1} .
$$

Therefore,

$$
\mathcal{P}(t)=e^{-\lambda t}[\mathcal{P}(0)-\mathcal{P}(\infty)]+\mathcal{P}(\infty),
$$

with $\lambda=-\ln \alpha$. In Fig. 2, $\mathcal{P}(\infty)$ is plotted for $\mu=4, \nu=2$.

\section{Purity statistics}

Equation (34) suggests that the average system purity is the same one would obtain from a partition of a random state of a Hilbert space of dimension $\mu(\mu \nu)$, i.e., Eq. (34) coincides with Lubkin's result if we assume that the system qubit
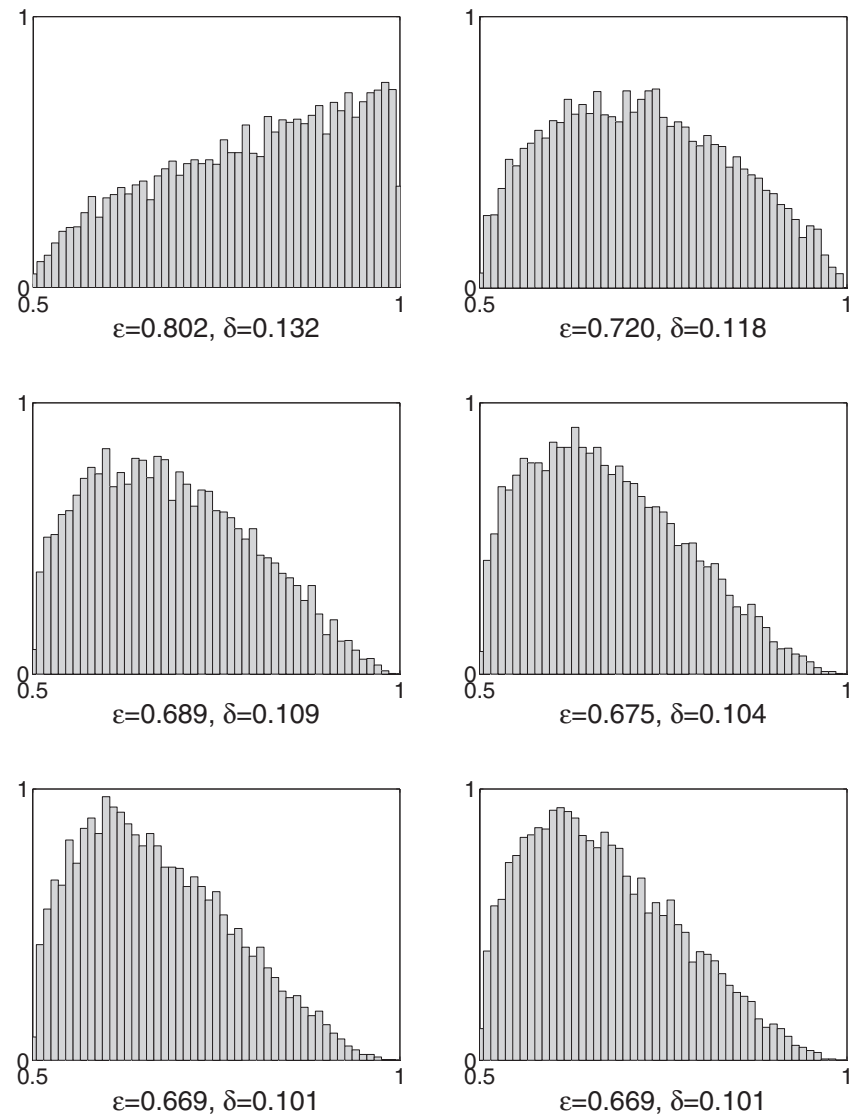

FIG. 3. Histograms showing the statistical distribution of the purities of the qubit system for $\mu=2, \nu=2$. Each histogram is obtained by considering a numerically generated large number of sequences of random collisions. From top to bottom, reading from left to right, the statistical distribution of the system purity after the first, the second,....,the sixth collision. In the lower side of each histogram are reported the values of the average $(\varepsilon)$ and of the standard deviation $(\delta)$ of each distribution. Note the agreement between the numerical average values of the purities and their ensemble-averaged values shown in Fig. 2.

has interacted with an environment of effective size $\nu_{e}=\mu \nu$. To support this conjecture we have plotted in Fig. 3 the numerically generated histograms of the statistical distributions of the system purities for each of the first six collisions for the case of colliding qubits. Indeed, as shown in Fig. 4, after just six collisions the histogram basically coincides with the purity distribution of a system qubit colliding with a single qudit of dimension 4.

An intuitive explanation of such behavior can be given in simple terms. After the first collision between system and environment qudits the system becomes mixed-even if originally it was in a pure state. Following a standard mathematical procedure however its state can be purified by introducing a fictitious qudit of dimension $\mu$ entangled with the system qudit. Therefore the whole process can be seen as a sequence of collisions among three qudits - the third one being the fictitious purification qudit-in an overall pure state. There is evidence [6] that in this case the overall system will evolve into a pure random state in a Hilbert space of 


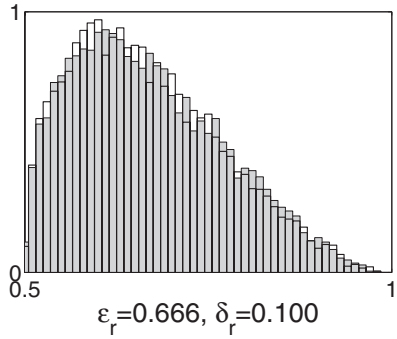

FIG. 4. Histogram comparison. Gray shadow: system purity distribution after the sixth collision (after which the equilibrium distribution is in practice already reached). Black: purity distribution of a system qubit colliding with a single qudit of dimension $\nu=4$, with average $\epsilon_{r}=0.666$ and variance $\delta_{r}=0.1000$.

dimension $\mu^{2} \nu$. This explains the purity statistics of the system qudit.

We have further numerical evidence to support the above analysis: in [34] Scott and Caves reported an analytical expression of the variance of the system purity distribution for an overall random state of two subsystems of dimensions respectively $\mu$ and $\nu$. This variance turns out to be

$$
\Delta_{S C}^{2} \mathcal{P}=\frac{2\left(\mu^{2}-1\right)\left(\nu^{2}-1\right)}{(\mu \nu+3)(\mu \nu+2)(\mu \nu+1)^{2}} .
$$

In our case instead the numerically calculated variance of the system purity distribution at equilibrium turns out to be well described by the above formula if we make the substitution $\nu \rightarrow \nu_{e}=\nu \mu$, i.e.,

$$
\Delta_{\infty}^{2} \mathcal{P}=\frac{2\left(\mu^{2}-1\right)\left(\nu_{e}^{2}-1\right)}{\left(\mu \nu_{e}+3\right)\left(\mu \nu_{e}+2\right)\left(\mu \nu_{e}+1\right)^{2}} .
$$

In Table I we report the standard deviations for different values of $\mu$ and $\nu$. In the second column we report the value of the Scott and Caves modified standard deviations of Eq. (39), while in the third column we report the corresponding numerically computed standard deviation values. Indeed a very good agreement between the two sets of values is clearly seen.

\section{ENTANGLEMENT DYNAMICS}

To further characterize the approach to equilibrium, in this section we illustrate some aspects of the entanglement dy-

TABLE I. Comparison between the Scott and Caves modified standard deviations $\left(\mu, \nu \rightarrow \mu, \nu_{e}=\nu \mu\right)$ and numerically calculated standard deviations.

\begin{tabular}{lcc}
\hline \hline$\mu, \nu$ & Scott and Caves & Collision model \\
\hline$\mu=2, \nu=2$ & 0.1005 & 0.1010 \\
$\mu=2, \nu=3$ & 0.0769 & 0.0767 \\
$\mu=3, \nu=2$ & 0.0608 & 0.0628 \\
$\mu=4, \nu=2$ & 0.0382 & 0.0388 \\
$\mu=2, \nu=4$ & 0.0618 & 0.0632 \\
$\mu=3, \nu=3$ & 0.0433 & 0.0438 \\
\hline \hline
\end{tabular}

namics for qubits, i.e., $\mu=\nu=2$. Since the overall state of the system and chain remains pure, the entanglement dyanamics is conveniently characterized in terms of the so-called tangles $[32,33]$. We remind the reader that, given the density operator $\rho_{i j}$ of a bipartite system of two qubits, the tangle $\tau_{i \mid j}$ is defined as

$$
\tau_{i \mid j}(\rho)=\left[\max \left\{0, \alpha_{1}-\alpha_{2}-\alpha_{3}-\alpha_{4}\right\}\right]^{2},
$$

where $\left\{\alpha_{k}\right\}(k=1, \ldots, 4)$ are the square roots of the eigenvalues (in nonincreasing order) of the non-Hermitian operator $\bar{\rho}_{i j}=\rho_{i j}\left(\sigma_{y} \otimes \sigma_{y}\right) \rho_{i j}^{*}\left(\sigma_{y} \otimes \sigma_{y}\right), \sigma_{y}$ is the $y$ Pauli operator, and $\rho_{i j}^{*}$ is the complex conjugate of $\rho_{i j}$, in the eigenbasis of the $\sigma_{z} \otimes \sigma_{z}$ operator. The concurrence $C$ is defined simply as $C_{i j}=\sqrt{\tau_{i \mid j}}$. The tangle $\tau_{i \mid j}$, or equivalently the concurrence $C_{i j}$, can be used to quantify the entanglement between the pair of qubits $i, j$ for an arbitrary reduced density operator $\rho_{i j}$. In our case the overall state of the system and the chain is pure. Therefore, the amount of entanglement between qubit $i$ and all the remaining qubits can be quantified by the tangle $\tau_{i \mid \text { rest }}=4 \operatorname{det} \rho_{i}$. After $t$ collisions, the tangle $\tau_{0 \mid \text { chain }}(t)$ between the system qubit and the chain conveys the same information as the purity $\mathcal{P}(t)$. Indeed, it is easy to show that

$$
\tau_{0 \mid \text { chain }}(t)=2-2 \mathcal{P}(t) .
$$

The purely multipartite entanglement $\tau_{M}$ established between the system qubit and the qubits of the chain can be quantified as

$$
\tau_{M}(t)=\tau_{0 \mid \text { chain }}(t)-\sum_{j=0}^{t} \tau_{0 \mid j},
$$

i.e., as the amount of entanglement which cannot be ascribed to purely bipartite entanglement between the system qubit and each individual environment qubit. Due to the complexity of the analytical expressions of the tangles, we resort to numerical simulations. Note that, in order to evaluate the tangle $\tau_{0 \mid \text { chain }}$ we have to numerically find the pairwise tangle between the system qubit and each qubit of the chain. This implies that we have to retain the overall system-chain density matrix; i.e., in contrast to the computation of the system purity $\mathcal{P}$, we cannot trace over the chain after each collision. In other words, as far as the entanglement dynamics is concerned, the qubit-chain model is not equivalent to a model in which the system qubit collides with a single environment qubit whose state is refreshed after each collision.

The pairwise tangles $\tau_{0 \mid j}$ are shown in Fig. 5, as a function of the number of collisions. A nonzero tangle $\tau_{0 \mid t^{\prime}}$ between the system qubit and the $t^{\prime}$ th qubit of the chain is generated immediately after the $t^{\prime}$ th collision and then quickly decays for $t>t^{\prime}$. The dependence of $\tau_{0 \mid t}(t)$ on the number $t$ of collisions is fitted satisfactorily by the exponential curve $\tau_{0 \mid t}(t)$ $=0.166+0.512 \exp (-0.921 t)$, suggesting the asymptotic value $\tau_{0 \mid \infty}(\infty)=0.166$ for the pairwise tangle generated between the system qubit and a qubit of the chain immediately after their collision.

Our numerical data shown in Fig. 5 also suggest that the weight of the terms $\tau_{0 \mid j}(t)$ with $j \neq t$ can be neglected with respect to $\tau_{0 \mid t}(t)$. Under this approximation and using Eqs. (42) and (41) we can estimate the asymptotic multipartite 


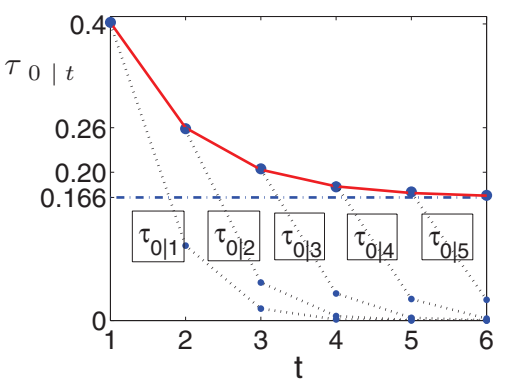

FIG. 5. (Color online) Pairwise tangles $\tau_{0 \mid j}$ as a function of the number $t$ of collisions (dashed curves with circles). The top circles refer to $\tau_{0 \mid t}(t)$ immediately after the $t$ th collision and are fitted by the solid curve $\tau_{0 \mid t}(t)-\tau_{0 \mid \infty}(\infty)=0.512 \exp (-0.921 t)$, with the fitting parameter $\tau_{0 \mid \infty}(\infty)=0.166$.

entanglement established between the system qubit and the qubits of the chain as

$$
\tau_{M}(\infty) \approx[2-2 \mathcal{P}(\infty)]-\tau_{0 \mid \infty}(\infty) \approx \frac{1}{2}
$$

This expectation is confirmed by the numerical data shown in Fig. 6: the convergence of $\tau_{M}(t)$ to its asymptotic value $\tau_{M}(\infty)$ is well fitted by the exponential decay $\tau_{M}(t)-\tau_{M}(\infty)$ $=-1.491 \exp (-0.849 t)$, with the fitting parameter $\tau_{M}(\infty)$ $=0.472 \approx \frac{1}{2}$.

\section{CONCLUSIONS}

We have investigated the dynamics of a system qudit of dimension $\mu$ sequentially interacting with the qudits, of di-

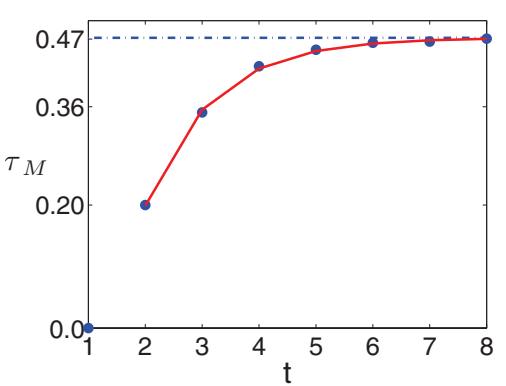

FIG. 6. (Color online) Multipartite entanglement $\tau_{M}$ for the system qubit as a function of the number $t$ of collisions. The solid line represents the exponential fit $\tau_{M}(t)-\tau_{M}(\infty)=-1.491 \exp (-0.849 t)$, with the fitting parameter $\tau_{M}(\infty)=0.472$.

mension $\nu$, of a chain. Each pairwise collision is modeled as a random transformation drawn from the Haar measure on $\mathrm{U}(\mu \nu)$. The relaxation to equilibrium, in terms of the ensemble average over random collisions, is analytically investigated by means of a Markov chain approach. We have shown that the steady state is the one corresponding to the steady state for random collisions with a single environment qudit of effective dimension $\nu_{e}=\nu \mu$. Furthermore, in contrast to the case of the homogenization process induced by purely elastic partial swap collisions [3], random unitary collisions can generate multipartite entanglement.

\section{ACKNOWLEDGMENTS}

G.G. and G.M.P. acknowledge support from PRIN "Quantum Noise in Mesoscopic Systems" and from EUROTECH S.p.A.
[1] V. Scarani, M. Ziman, P. Štelmachovič, N. Gisin, and V. Bužek, Phys. Rev. Lett. 88, 097905 (2002).

[2] M. Ziman, P. Štelmachovič, V. Bužek, M. Hillery, V. Scarani, and N. Gisin, Phys. Rev. A 65, 042105 (2002).

[3] M. Ziman, P. Štelmachovič, and V. Bužek, J. Opt. B: Quantum Semiclassical Opt. 5, S439 (2003); Open Syst. Inf. Dyn. 12, 81 (2005); M. Ziman and V. Bužek, Phys. Rev. A 72, 022110 (2005).

[4] M. Koniorczyk, A. Varga, P. Rapčan, and V. Bužek, Phys. Rev. A 77, 052106 (2008).

[5] G. Benenti and G. M. Palma, Phys. Rev. A 75, 052110 (2007).

[6] G. Gennaro, G. Benenti, and G. M. Palma, Europhys. Lett. 82, 20006 (2008).

[7] P. Diaconis, Not. Am. Math. Soc. 52, 11 (2005).

[8] F. Mezzadri, Not. Am. Math. Soc. 54, 592 (2007).

[9] M. Kus and K. Życzkowski, Phys. Rev. A 44, 956 (1991).

[10] K. Życzkowski and M. Kus, J. Phys. A 27, 4235 (1994).

[11] K. Życzkowski and M. Kus, Phys. Rev. E 53, 319 (1996).

[12] M. Pozniak, K. Życzkowski, and M. Kus, J. Phys. A 31, 1059 (1998).

[13] C. Pineda and T. H. Seligman, Phys. Rev. A 75, 012106 (2007); C. Pineda, T. Gorin, and T. H. Seligman, New J. Phys. 9, 106 (2007)

[14] A. Akhalwaya, M. Fannes, and F. Petruccione, J. Phys. A:
Math. Theor. 40, 8069 (2007).

[15] J. Emerson, Y. S. Weinstein, M. Saraceno, S. Lloyd, and D. G. Cory, Science 302, 2098 (2003).

[16] J. Emerson, in Quantum Communication, Measurement, and Computing, edited by S. M. Barnett, E. Andersson, J. Jeffers, P. Öhberg, and O. Hirota, AIP Conf. Proc. No. 734 (AIP, Melville, NY, 2004).

[17] J. Emerson, E. Livine, and S. Lloyd, Phys. Rev. A 72, 060302(R) (2005).

[18] Y. S. Weinstein and C. S. Hellberg, Phys. Rev. Lett. 95, 030501 (2005).

[19] O. C. O. Dahlsten, R. Oliveira, and M. B. Plenio, J. Phys. A: Math. Theor. 40, 8081 (2007)

[20] R. Oliveira, O. C. O. Dahlsten, and M. B. Plenio, Phys. Rev. Lett. 98, 130502 (2007).

[21] M. Žnidarič, Phys. Rev. A 76, 012318 (2007).

[22] D. Rossini and G. Benenti, Phys. Rev. Lett. 100, 060501 (2008).

[23] G. Benenti, e-print arXiv:0807.4364v1.

[24] M. Žnidarič, Phys. Rev. A 78, 032324 (2008).

[25] A. Harrow, P. Hayden, and D. Leung, Phys. Rev. Lett. 92, 187901 (2004).

[26] C. H. Bennett, P. Hayden, D. Leung, P. Shor, and A. Winter, IEEE Trans. Inf. Theory 51, 56 (2005). 
[27] P. Hayden, D. Leung, P. Shor, and A. Winter, Commun. Math. Phys. 250, 371 (2004).

[28] P. Hayden, D. W. Leung, and A. Winter, Commun. Math. Phys. 265, 95 (2006).

[29] E. Lubkin, J. Math. Phys. 19, 1028 (1978).

[30] J. Schlienz and G. Mahler, Phys. Rev. A 52, 4396 (1995).
[31] D. Gottesman, Chaos, Solitons Fractals 10, 1749 (1999).

[32] W. K. Wootters, Phys. Rev. Lett. 80, 2245 (1998).

[33] V. Coffman, J. Kundu, and W. K. Wootters, Phys. Rev. A 61, 052306 (2000).

[34] A. Scott and C. Caves, J. Phys. A 36, 9553 (2003). 